\title{
Correlation of Optical Coherence Tomographic Findings with Clinical Features in High Myopia
}

\author{
Evangeline Rao ${ }^{1}$ Maithili Mishra ${ }^{1}$ Sheela Kerkar \\ ${ }^{1}$ Department of Ophthalmology, King Edward Memorial Hospital, \\ Acharya Donde Marg, Parel, Mumbai, Maharashtra, India
}

\begin{abstract}
Address for correspondence Evangeline Rao, MBBS, MS, FICO, King Edward Memorial Hospital, Department of Ophthalmology, Acharya Donde Marg, Parel, Mumbai 400012, Maharashtra, India (e-mail: evangeline.rao@gmail.com).
\end{abstract}

Int J Recent Surg Med Sci 2019;5:19-25
Abstract
Keywords
- high myopia
- optical coherence tomography
- macular thickness
- retinal nerve fiber layer thickness
- axial length

This is a cross-sectional observational study to correlate relationship between macular and retinal nerve fiber layer (RNFL) thickness in relation to clinical features in high myopia. A total of 100 eyes of 50 consecutive patients underwent optical coherence tomography (OCT) of the macula and RNFL. It was observed that correlation of RNFL thickness to the axial length is better than that of RNFL thickness to the spherical equivalent. The macular thickness in the parafoveal region was observed to be thicker than the perifoveal region in all quadrants. This study therefore emphasizes the need to have macular thickness nomogram for high myopes to avoid misinterpretation of OCT results due to axial length and refractive error and, also, the need for a routine baseline OCT scan for all high myopia patients.

\section{Introduction}

Refractive errors are found to be the most common cause of visual impairment and second major cause of avoidable blindness in India. ${ }^{1}$ Myopia is a leading cause of visual disability throughout the world. It adds to the vision challenges because high myopia increases the risk of pathologic ocular changes such as cataract, glaucoma, retinal detachment, and myopic macular degeneration, which can cause irreversible vision loss. ${ }^{2}$ Over the past few decades, there has been a steady increase in the prevalence of myopia in some populations, especially South East Asia, leading to growing concern among the public and the scientific community. In communities with a high prevalence of pathologic myopia, myopic macular degeneration has been found to be the most frequent cause of irreversible blindness. ${ }^{3}$

High myopia is defined as a refractive error of -6.00 diopters (D) or greater. It has also been defined on the basis of axial length exceeding $26 \mathrm{~mm} .{ }^{4}$ Individuals with high myopia are subject to a variety of characteristic pathologic changes such as tessellated fundus, lacquer cracks, patchy or diffuse atrophy, choroidal neovascular (CNV) membrane, posterior staphyloma, straightened and stretched vessels, temporal peripapillary atrophic crescent, hemorrhages, and tilting of the optic disc. According to histologic studies, the retina becomes atrophic and degenerates in myopic

received

March 1, 2019

accepted after revision

March 25, 2019 eyes, particularly at the posterior pole, and these changes are associated with a high frequency of macular abnormalities. ${ }^{5}$ These findings consist of dehiscence of retinal layers known as retinoschisis, paravascular inner retinal cysts and lamellar holes, peripapillary intrachoroidal cavitation (also known as peripapillary detachment in pathologic myopia), tractional internal limiting membrane (ILM) detachment, macular holes (lamellar and full thickness), posterior retinal detachment, and CNV membranes. ${ }^{4}$

Traditional methods of evaluating macular and retinal nerve fiber layer (RNFL) thickness including slit lamp biomicroscopy, ultrasonography, and fundus photography are not accurately quantitative. They are relatively insensitive to minute changes in retinal thickness and also unable to detect specific anatomic details, especially at vitreomacular interface. $^{6}$

Optical coherence tomography (OCT) is a diagnostic imaging technology that can perform micrometer resolution cross-sectional or tomographic imaging of macula and retinal layers. It is a noninvasive technique that measures retinal thickness using time delays of reflected or backscattered light and interferometry. ${ }^{7.8}$ OCT with its fine cross-sectional imagery of retinal structures has tremendously facilitated the evaluation of posterior vitreoretinal anatomy in eyes with high myopia. It can detect otherwise undetectable retinal changes in asymptomatic patients. ${ }^{4}$

C2019 Medical and Surgical

Update Society
License terms

(이 (1) $\Theta \circledast$ 
The Stenstroms study in Uppsala, Sweden showed that $29 \%$ of the general population have low myopia $(<-2 \mathrm{D})$, $7 \%$ have moderate myopia (2-6 D), and $2.5 \%$ have high myopia (>6D). Studies from the early 2000s from India have quoted prevalence figures of myopia as $7.4 \%$ by Murthy et al and $4.1 \%$ by Dandona et al. ${ }^{1}$ Various surveys in India have found myopia prevalence ranging from 6.9 to $19.7 \%{ }^{1}$ The World Health Organization (WHO) estimates 2.5 billion nearsighted people by 2020 . Based on the data available for prevalence of high myopia and using the formula $n=Z^{2} P$ $(1-P) / d^{2}$, where $P=2.5 \%$ (prevalence of high myopia obtained from the Stenstroms study), $d=5 \%$, and $Z=1.96$, the sample size has been calculated as 50 patients.

\section{Objectives}

The aims of the study are to correlate clinical signs in high myopia such as spherical equivalent (SE) and axial length to macular and RNFL thickness. The objective of the study is to evaluate the role of OCT in detection of minor and major retinal changes that later may lead to irreversible vision loss in high myopic eyes that are missed on routine ophthalmic examination.

\section{Methods}

It is a cross-sectional population based observational study wherein 100 eyes of 50 high myopic patients were analyzed. The study was conducted in compliance with the institutional ethical committee and study protocols as well as the ICMR guidelines for biomedical research in human subjects, 2006.

The inclusion criteria were patients of either sex, aged between 18 and 50 years, with high myopia (patients having spherical equivalence above -6 spherical diopter or having axial length of $>26 \mathrm{~mm}$ ), and willing for OCT evaluation and complying with protocol requirements.

The exclusion criteria were patients with other ocular pathologies unrelated to myopia affecting vision, those who have undergone any other ocular surgeries besides uncomplicated cataract surgery, those who have undergone ocular laser treatment for any ocular pathology, and intraocular pressure greater than $21 \mathrm{~mm} \mathrm{Hg}$.

Patients with high myopia were recruited from the outpatient department of ophthalmology of a tertiary care centre. Fifty consecutive high myopes fulfilling the above criteria were enrolled for the study, and appropriate written informed consents were taken. Detailed ocular and systemic history was obtained; complete routine ophthalmologic examination including retinoscopy, indirect ophthalmoscopy, slitlamp biomicroscopy including 78/90D fundus examination, and A-scan for axial length measurement was done; and patients not fulfilling inclusion criteria were excluded from the study. OCT for macular and RNFL thickness was performed on the included study patients.

OCT examination was done through a dilated pupil using commercially available spectral domain OCT system (Cirrus HD-OCT Model 500-Carl Zeiss Meditec, Inc., Dublin, California, United States). The calculation of macular thickness was based on the 6-mm retinal thickness map analysis printout. The map was composed of nine sectoral thickness measurements in three concentric circles with diameters of 1,3 , and $6 \mathrm{~mm}$. The central $1-\mathrm{mm}$ circular region represented the fovea. The area bounded by the outer $(6 \mathrm{~mm})$ and middle $(3 \mathrm{~mm}$ ) circles formed the outer ring (perifovea) and the area bounded by middle $(3 \mathrm{~mm}$ ) and inner circles $(1 \mathrm{~mm})$ formed the inner ring (parafovea). The perifovea and parafovea were further divided into four quadrantic zones: temporal, superior, nasal, and inferior. The peripapillary scan was divided into four equal 90-degree quadrants (superior, inferior, temporal, nasal), and RNFL thickness in these four quadrants was measured.

\section{Results}

This study was conducted on 100 eyes of 50 high myopic patients consisting of 28 female subjects and 22 male subjects. Among the participants 56\% were female and $44 \%$ were male patients. The participants were aged between 19 and 50 years with the mean age of $31.96 \pm 9.81$ years. Their SE ranged from -6 to -23.25 diopters with the mean SE of $-10.94 \pm 4.79 D$. Their axial length ranged from 23.25 to $35.1 \mathrm{~mm}$ with the mean axial length of $26.24 \pm 2.29 \mathrm{~mm}$.

The mean RNFL thickness in the inferior quadrant was observed to be $87.70 \pm 24.06 \mu \mathrm{m}$ that was greater than the superior quadrant $(85.95 \pm 21.01 \mu \mathrm{m})$, followed by the nasal quadrant $(59.11 \pm 14.50 \mu \mathrm{m})$ and then the temporal quadrant (58.81 $\pm 15.62 \mu \mathrm{m})$. Thus, our results showed the characteristic "double hump pattern" and followed the "ISNT rule" that states that the inferior neuroretinal rim is the broadest followed by the superior, nasal, and temporal neuroretinal rim.

The RNFL thickness was found to have a positive correlation with SE, which was statistically significant in the superior, nasal, and inferior quadrants whereas in the temporal quadrant RNFL thickness did not show statistically significant correlation with SE. The highest correlation was found in the inferior quadrant with coefficient of correlation of $0.58412(p=0)$. In other words, the RNFL thickness decreases with the degree of myopia based on spherical equivalence.

The RNFL thickness was observed to have a negative correlation with the axial length of the eyeball, which was statistically significant in the superior, nasal, and inferior quadrants. However, the negative correlation between RNFL thickness and axial length of the eyeball in the temporal quadrant was not statistically significant. The highest negative correlation was found in the inferior quadrant with coefficient of correlation $(r)$ of $-0.5494(p=0)$.

The mean central macular thickness (CMT) foveal thickness of patients in our study was observed to be 227.41 $\pm 35.61 \mu \mathrm{m}$. The parafoveal region was observed to be thicker than the perifoveal region in all quadrants. The nasal quadrant was observed to be thickest followed by superior quadrant, inferior quadrant, and then the temporal quadrant in both the parafoveal and perifoveal regions. The mean nasal parafoveal thickness was calculated as $299.50 \pm 28.03 \mu \mathrm{m}$. 
The mean superior parafoveal thickness was calculated as $298.03 \pm 24.17 \mu \mathrm{m}$. The mean inferior parafoveal thickness was found to be $294.63 \pm 25.90 \mu \mathrm{m}$. The mean temporal parafoveal thickness was observed to be $286.89 \pm 24.61 \mu \mathrm{m}$. The mean nasal perifoveal thickness was calculated as 269.05 $\pm 35.01 \mu \mathrm{m}$. The mean superior perifoveal thickness was seen to be $255.66 \pm 33.21 \mu \mathrm{m}$. The mean inferior perifoveal thickness was $248.01 \pm 27.50 \mu \mathrm{m}$. The mean temporal perifoveal thickness was $243.07 \pm 30.05 \mu \mathrm{m}$.

The macular thickness was found to have a statistically significant positive correlation with SE in all the macular subfields with the highest coefficient of correlation $(r)$ of $0.52548(p=0)$ in the temporal parafoveal region. The macular thickness was observed to have a statistically significant negative correlation with the axial length of the eyeball in all the macular subfields with the highest negative coefficient of correlation $(r)$ of $-0.49587(p=0)$ in the superior parafoveal subfield.

Analysis of the collected data was done using Graphpad statistical software. The correlation between RNFL thickness and macular thicknesses with axial length and SE were examined and expressed as the Spearman's coefficient of correlation $(r)$. $p$-Value of $\leq 0.05$ was considered statistically significant.

\section{Discussion}

This study was conducted on 100 eyes of high myopes wherein the mean RNFL in the inferior quadrant was greater than the superior quadrant followed by the nasal quadrant and then the temporal quadrant. In the previously described literature by Lee et al, Leung et al, and Malakar et al, RNFL thickness demonstrated characteristic "double hump pattern" and followed by the "ISNT rule" that states that the inferior neuroretinal rim is the broadest followed by the superior, nasal, and temporal neuroretinal rim as given by Jonas et al. ${ }^{10}$ These observations are supported by Parvaresh et $\mathrm{al}^{11}$ and by histologic data as well. Our study also found that temporal RNFL to be thinner than the nasal RNFL. However, Schumann et al ${ }^{12}$ and Bowd et $\mathrm{al}^{13}$ report that the RNFL in the nasal quadrant was found to be thinner than the temporal quadrant in their studies. ${ }^{9}$

Several prior studies such as the Garcia et al, ${ }^{14}$ Hoh et al,,${ }^{15}$ Kashiwagi et $a{ }^{16}$ and Melo et al ${ }^{17}$ did not find any significant association between myopia and RNFL thickness. These studies may have been limited by the poorer resolution of earlier-generation OCT and confocal laser devices, and thus lower sensitivity. Indeed, histologic measurements of the thickness of the RNFL in normal eyes demonstrate that superior, inferior, and nasal quadrant RNFL at the disc margin are significantly thicker than the temporal quadrant RNFL at the disc margin. This probably results from subtle differences in axon trajectory away from the optic nerve head $(\mathrm{ONH})$ and emphasizes the importance of eccentricity when comparing RNFL thickness around the ONH. ${ }^{9}$ The difference between the above two studies is attributed to population characteristics and measurement techniques. Malakar et $\mathrm{al}^{9}$ observed that RNFL thickness showed double-hump pattern in both myopes and emmetropes. This study also stated that emmetropic eyes are consistently spherical, but myopic eyes demonstrated symmetrical or asymmetrical anteroposterior elongation and posterior protrusions. They postulated that the distribution of the RNFL bundles conforms to the eyeball shape, which leads to double hump pattern.

The RNFL thickness was found to have a positive correlation with SE, which was statistically significant in the superior, nasal, and inferior quadrants whereas in the temporal quadrant RNFL thickness did not show statistically significant positive correlation with SE. The retinal nerve fiber layer thickness decreases with the degree of myopia (i.e., more negative spherical equivalence).

The RNFL thickness was observed to have a negative correlation with the axial length of the eyeball, which was statistically significant in the superior, nasal, and inferior quadrants.

Thus, increased axial length appears to decrease the RNFL and macular thickness more in the nasal area.

Bendschneider et $\mathrm{al}^{18}$ found significant association between axial length, refractive error, and RNFL thickness. Rauscher et $\mathrm{al}^{19}$ observed decrease in RNFL thickness with axial length $(7 \mu \mathrm{m} / \mathrm{mm})$ was higher than previously published for other populations (Budenz et al: $2 \mu \mathrm{m} / \mathrm{mm},{ }^{20}$ Leung et al: $2.75 \mu \mathrm{m} / \mathrm{mm},{ }^{21}$ Salchow et al: $1.7 \mu \mathrm{m} / \mathrm{mm}^{22}$ ). They stated that thin RNFL correlated more strongly with axial length than with SE, because population variations in the refractive power of the cornea and lens could affect refractometry (but likely not the axial length). Therefore, stronger correlation with axial length indicates that globe elongation (with geometrically concomitant thinning of the globe wall) is an important determinant of thin RNFL.

Our study observed a similar pattern of correlation, wherein longer the axial length of an eye, thinner is the RNFL as compared to correlation of SE with RNFL thickness in the same eye $(p<0.0059)$. Thin RNFL could be attributed to an elongation and thinning of the retina and sclera, which would spread the nerve fibers over a larger surface area. ${ }^{21}$ It could also represent, in part, an actual decrease in nerve fiber number, although this does not appear to be the case based on histologic studies. ${ }^{23}$ Parvaresh et al, ${ }^{11}$ found strong negative correlation between RNFL thickness and AL $(r-0.91, p=0.003)$ that could be explained by the fact that the number of axons that forms the RNFL is equal and not proportional to AL. On the other hand, larger AL causes larger surface area, and distribution of an equal amount of retinal nerves in larger area causes a thinner layer. Leung et $\mathrm{al}^{21}$ as with our study, demonstrated the reduction in RNFL thickness with increasing axial length could be explained by the observation that there is increased sclera and retinal thinning in myopia. ${ }^{23}$

In myopic eyes, the elongation of the globe leads to mechanical stretching and thinning of the retina. Therefore, it is conceivable that the extent of the elongation would be related to the degree of retinal thinning, although it is yet to be ascertained whether the RNFL thickness is decreased at the histological level. Although the mechanisms responsible 
for the association between glaucoma and myopia are poorly understood, it has been postulated that the $\mathrm{ONH}$ in myopic eyes are structurally more susceptible to glaucomatous damage because of the changes in connective tissue structure and arrangement. ${ }^{23}$ As the integrity of RNFL is a recognized surrogate for glaucomatous change, the finding of decreasing RNFL thickness with increasing myopia supports this conclusion of the Blue Mountain eyes study. ${ }^{19}$

The mean CMT (foveal thickness) of our study was observed to be $227.41 \pm 35.61 \mu \mathrm{m}$. The parafoveal region was observed to be thicker than the perifoveal region in all quadrants. The nasal quadrant was observed to be thickest, followed by superior quadrant, inferior quadrant and then the temporal quadrant in both the parafoveal and perifoveal regions. The macular thickness was found to have a statistically significant positive correlation with SE in all the macular subfields, with the highest coefficient in the temporal parafoveal region. The macular thickness was observed to have a statistically significant negative correlation with the axial length of the eyeball in all the macular subfields, with the highest negative coefficient of correlation superior parafoveal subfield.

Appukuttan et $\mathrm{al}^{24}$ Tewari et al, ${ }^{25}$ and Massin et $\mathrm{al}^{26}$ stated that refraction was not found to have any significant effect on macular thickness. However, Lim et $\mathrm{al}^{35}$ in their study on myopes, found that myopes had thinner parafovea and thicker foveas. They suggested that axial myopia may result in a flattening, and perhaps "thinning out" of the retinal layers, including the nerve fiber layer; however, they found that overall macular thickness outside the fovea was not associated with axial length. Wu et $\mathrm{al}^{23}$ also observed that the retinal thickness in high myopia is greater in the foveola and fovea, but significantly lesser in the inner and outer macular region. They stated that retina in highly myopic individuals has smaller macular volume than that of nonmyopic individuals. We propose that this phenomenon may be due to the increased axial length of the enlarged eyeball, resulting in mechanical stretching of the sclera. Under this condition, retinal stretching would also occur with panretinal thinning. On the other hand, the stretching and flattening tendency of the ILM and the centripetal force of the posterior vitreous result in elevation of the foveola and fovea leading to greater thickness at the fovea. With age, in addition to mechanical stretching of the eyeball, these factors also play an important role in the development of myopic fundus changes. ${ }^{23}$ The elevated foveola and fovea area in young, healthy highly myopic eyes might explain the high incidence of macular hole, which may be associated with retinal detachment, myopic traction maculopathy, and foveoschisis. ${ }^{23,27}$

Zhao et $\mathrm{al}^{5}$ found that different macular parts, as well as different macular layers, changed in different manners. Whereas foveal thickness was not correlated with AL or SE, the para- and perifoveal thicknesses were negatively correlated with AL and, positively, correlated with SE. These findings were consistent with the results by Wakitani, Lim, ${ }^{9}$ and Liu, ${ }^{28}$ suggesting that retinal thinning in myopia occurred more frequently in the peripheral part. Lam et $\mathrm{al}^{29}$ recently found that, at the outer ring (3-6 $\mathrm{mm}$ ) and not at the inner ring $(1-3 \mathrm{~mm})$, macular thickness decreased in myopia. Similar observation was made in our study in which parafoveal (inner macula) region was thicker than perifoveal (outer macula) region. Increased foveal thickness in myopic eyes has been reported in other studies as well. ${ }^{5}$ Tangential traction by the ILM or posterior vitreous cortex was hypothesized to be one of the reasons, whereas Luo suggested that increased foveal thickness might be caused by the high permeability of the RPE at the fovea. ${ }^{5}$ Zhao et $a l^{5}$ found that increasing retinal thickness occurred predominantly in the inner layer, which appeared to support the theory of traction acting on the inner surface.

The study by Waris et $\mathrm{al}^{30}$ found that the overall mean macular thickness was significantly less in all age group of myopic eyes (group I) as compared with emmetropic eyes (group II). However, in contrast, the fovea (central zone) was highly significantly thicker $(p<0.0001)$ in myopic eyes as compared with emmetropic eyes (group II). The study by Waris et al found that the parafoveal area (inner ring) retinal thickness was very highly significantly thin in myopic eyes as compared with emmetropic eyes. The comparison of a parafoveal zone with other parafoveal zones in myopes revealed the parafoveal area of macula in nasal quadrant was the thickest and temporal quadrant was thinnest. ${ }^{30}$ This finding was similar in our study.

Sung-Won Choi et al ${ }^{31}$ conducted a study in Korean adults and Marcus C. C. Lim et al ${ }^{32}$ conducted a study in Asians, and they observed foveal thickness increased with myopia. A study by Dennis Shun Chiu Lam et al ${ }^{33}$ in Chinese population in 2005 found that the average foveal $(1 \mathrm{~mm})$ thickness was significantly higher in the high myopes than low to moderate myopes $(p=0.002)$.

In an OCT macular thickness study, Wakitani et al ${ }^{34}$ proposed that the periphery, rather than the central, retina is thinner in myopic eyes. The absence of large blood vessels and optic nerve fibers could render the peripheral retina less resistant to traction and stretch, and the decrease in peripheral retinal thickness may compensate for the stretching force over the entire retina to preserve the central retinal thickness. This conclusion is supported by Lim et $\mathrm{al}^{35}$ who also suggested that retinal thinning in myopia is more common in the peripheral retina. They found that the outer ring (3-6 mm), but not the inner ring (1-3 mm), macular thicknesses decreased in eyes with a greater degree of myopia.

Mrugacz et $\mathrm{a}^{36}$ who showed that as myopia increased, the thickness of fovea decreased while the thickness of peripapillary RNFL remained unchanged. However, in our study we found that the peripapillary RNFL thickness also decreased with myopia along with the macular thickness.

Waris et $\mathrm{a}^{30}$ showed that para- and perimacular thickness was significantly less in high myopic, but central (foveal) thickness was greater in myopes. Liu et $\mathrm{al}^{28}$ stated that with an increase in axis length, macular thickness becomes thinner, especially in the outer retinal subfields. Marcus et $\mathrm{a}^{32}$ and Lim et $\mathrm{al}^{35}$ also reported that the parafovea was thinner in myopic patients. This observation may be the result of the anatomical features of the retinal macular foveolar region. The foveolar is the thinnest area of the retina, which consists of the five 
innermost cell layers of the retina, including the outer plexiform layer, outer nuclear layer, external limiting membrane, photoreceptor layer, and retinal pigment epithelium. Previous histopathologic studies reported that the thickness of neurosensory retina (RNL), especially the inner nuclear layer, was thinner in several animal models of myopia. ${ }^{28}$ The foveola lacks the innermost retinal layers, and there is no inner nuclear layer, so the retinal thickness variation is not obvious between the high myopia group and moderate myopia groups. Therefore, the macular retinal thickness in the parafovea was thinner in the high myopia group than in the moderate myopia group, but no difference was found in the foveola. However, in our study, central macular thinning was observed in patients of high myopia as well. This may be attributed to the fact that our study included both myopic patients with poor vision along with myopic patients with good vision. Hence, posterior staphyloma and other pathologic processes, such as choroid retinal degeneration, and choroid and RPE atrophy, could likewise play a role in changes of the retina. These characteristics can be used as an auxiliary diagnosis for making a distinction with other macular retinal related diseases. The possibility of poorer fixation with high myopia arose, as fixation not through the fovea would produce overestimated foveal thicknesses. Off-foveola fixation may have resulted in larger foveolar thickness measurements in other studies.

\section{Conclusion}

In conclusion, when the macular thickness is abnormal, clinicians should consider the effect of the refractive status of eye combined with clinical manifestations to comprehensively evaluate its clinical significance.

\section{Clinical Relevance Statement}

Individuals with high myopia are subject to various retinal pathologies including posterior pole and peripapillary lesions. Because of retinal thinning and chorioretinal changes in these persons, simple fundus examination may miss underlying retinal pathologies. OCT is an accurate tool that can localize such anatomical changes.

If the macular thickness in the foveola is abnormally thin, pathologic disease should be considered. However, if changes occurred in the parafovea, other factors can first be eliminated, such as refractive factors, which could influence the measurements of macular thickness by the OCT, especially among patients with high myopia.

Our study recommends obtaining a routine baseline high-definition OCT macular and retinal nerve fiber thickness scan, for all high myopic patients, in the context of their routine ophthalmologic examination to detect early changes to prevent vision threatening complications. This study therefore emphasizes the need to have macular thickness nomogram for high myopes for a given population group to avoid wrong interpretation.

In the future, combined technology to measure axial length can be integrated in an OCT machine to minimize misinterpretation and have more accurate scans. Until then, we recommend careful interpretation of macular and RNFL data on moderate to highly myopic individuals (particularly those with axial length $>26 \mathrm{~mm}$ ) when utilizing the currently available OCT devices.

The recently developed IOL master 700 (Carl Zeiss Meditech AG, Jena, Germany) is the first swept source OCTbased biometry. It gives image-based measurements viewing the complete longitudinal section of the eye. It helps to detect irregular eye geometries such as lens tilt and poor fixation by imaging the fovea. ${ }^{37}$ There are few case reports of optical biometry identifying macular pathology prior to cataract surgery. However, the OCT images obtained on IOL master 700 are indistinct, and hence they require an additional standard OCT to confirm the pathology. This study emphasizes the need to provide improved imaging prior to cataract surgery, especially in highly myopic patients as they have higher incidence of macular pathology.

\section{Ethical Approval}

This study was performed in compliance with the World Medical Association Declaration of Helsinki on Ethical Principles for Medical Research Involving Human Subjects and was reviewed by Seth GSMC \& KEM Hospital institutional review board.

\section{Conflict of Interest}

None declared.

\section{References}

1 Saxena R, Vashist P, Menon V. Is myopia a public health problem in India? Indian J Community Med 2013;38(2):83-85

2 Wong TY, Ferreira A, Hughes R, Carter G, Mitchell P. Epidemiology and disease burden of pathologic myopia and myopic choroidal neovascularization: an evidence-based systematic review. Am J Ophthalmol 2014;157(1):9-25

3 Iwase A, Araie M, Tomidokoro A, Yamamoto T, Shimizu H, Kitazawa Y; Tajimi Study Group. Prevalence and causes of low vision and blindness in a Japanese adult population: the Tajimi study. Ophthalmology 2006;113(8):1354-1362

4 Faghihi H, Hajizadeh F, Riazi-Esfahani M. Optical coherence tomographic findings in highly myopic eyes. J Ophthalmic Vis Res 2010;5(2):110-121

5 Zhao Z, Zhou X, Jiang C, Sun X. Effects of myopia on different areas and layers of the macula: a Fourier-domain optical coherence tomography study of a Chinese cohort. BMC Ophthalmol 2015;15:90

6 Chan A, Duker JS. A standardized method for reporting changes in macular thickening using optical coherence tomography. Arch Ophthalmol 2005;123(7):939-943

7 Hee MR, Izatt JA, Swanson EA, et al. Optical coherence tomography of the human retina. Arch Ophthalmol 1995; 113(3):325-332

8 Huang D, Swanson EA, Lin CP, et al. Optical coherence tomography. Science 1991;254(5035):1178-1181

9 Malakar M, Askari SN, Ashraf H, Waris A, Ahuja A, Asghar A. Optical coherence tomography assisted retinal nerve fibre layer thickness profile in high myopia. J Clin Diagn Res 2015;9(2):NC01-NC03

10 Jonas JB, Budde WM, Panda-Joans S. Ophthalmoscopic evaluation of the optic nerve head. Surv Ophthalmol 1999;43:293-320. 
11 Parvaresh MM, Imani M, Bahmani-Kashkouli M, Soltan-Sanjari M. Optical Coherence Tomography-Measured Nerve Fiber Layer and Macular Thickness in Emmetropic, High-Myopic and High-Hyperopic Eyes. Iranian Journal of Ophthalmology. 2008;20(2):4-9

12 Schumann JS, Hee, MR, Puliofito CA, et al. Quantification of nerve fiber layer thickness in normal and glaucomatous eyes using optical coherence tomography. Arch Ophthalmol. 1995;113(5):586-596

13 Bowd C, Weinreb RN, Williams JM, Zangwill LM. The retinal nerve fiber layer [16] thickness in ocular hypertensive, normal and glaucomatous eyes with optical coherence tomography. Arch Ophthalmol. 2000;118:22-26

14 Garcia-Valenzuela E, Anderson NG, Pons M, Iezzi R. Retinal thickness by OCT in subjects with emmetropia, hyperopia, and myopia. Invest Ophthalmol Vis Sci 2002;43E-Abstract 2574

15 Hoh ST, Lim MC, Seah SK, et al. Peripapillary retinal nerve fiber layer thickness variations with myopia. Ophthalmology 2006;113:773-777

16 Kashiwagi K, Tamura M, Abe K, et al. The influence of age, gender, refractive error, and optic disc size on the optic disc configuration in Japanese normal eyes. Acta Ophthalmol Scand 2000;78:200-203

17 Melo GB, Libera RD, Barbosa AS, et al. Comparison of optic disk and retinal nerve fiber layer thickness in nonglaucomatous and glaucomatous patients with high myopia. Am J Ophthalmol 2006;142:858-860

18 Bendschneider D, Tornow RP, Horn FK. Laemmer R, Roessler $\mathrm{CW}$, Juenemann AG, et al. Retinal nerve fibre layer thickness in normals measured by spectral domain OCT. J Glaucoma 2010;19:475-82

19 Rauscher FM, Sekhon N, Feuer WJ, Budenz DL. Myopia affects retinal nerve fiber layer measurements as determined by optical coherence tomography. J Glaucoma 2009;18(7):501-505

20 Budenz DL, Anderson DR, Varma R, et al. Determinants of normal retinal nerve fiber layer thickness measured by Stratus OCT. Ophthalmology 2007; 114:1046-1052

21 Leung CK, Mohamed S, Leung KS, et al. Retinal nerve fiber layer measurements in myopia: an optical coherence tomography study. Invest Ophthalmol Vis Sci 2006;47(12):5171-5176

22 Salchow DJ, Oleynikov YS, Chiang MF, et al. Retinal nerve fiber layer thickness in normal children measured with optical coherence tomography. Ophthalmology 2006;113:786-791

$23 \mathrm{Wu}$ PC, Chen YJ, Chen CH, et al. Assessment of macular retinal thickness and volume in normal eyes and highly myopic eyes with third-generation optical coherence tomography. Eye (Lond) 2008;22(4):551-55569

24 Appukuttan B, Giridhar A, Gopalakrishnan M, Sivaprasad S. Normative spectral domain optical coherence tomography data on macular and retinal nerve fiber layer thickness in Indians. Indian J Ophthalmol 2014;62(3):316-321

25 Tewari HK, Wagh VB, Sony P, Venkatesh P, Singh R. Macular thickness evaluation using the optical coherence tomography in normal Indian eyes. Indian J Ophthalmol 2004; 52:199-204.

26 Massin P, Erginay A, Haouchine B, Mehidi AB, Paques M, Gaudric A. Retinal thickness in healthy and diabetic subjects measured using optical coherence tomography mapping software. Eur J Ophthalmol 2002;12:102-108

27 Panozzo G, Mercanti A. Optical coherence tomography findings in myopic traction maculopathy. Arch Ophthalmol 2004;122(10):1455-1460

28 Liu L, Zou J, Jia L, Yang JG, Chen SR. Spectral- and time-domain optical coherence tomography measurements of macular thickness in young myopic eyes. Diagn Pathol 2014;9:38

29 Lam DS, Leung KS, Mohamed S, Chan WM, Palanivelu MS, Cheung CY, et al. Regional variations in the relationship between macular thickness measurements and myopia. Invest Ophthalmol Vis Sci. 2007;48(1):376-82

30 Waris A, Malakar M, Askari SN, Ashraf H, Asagar A. Optical coherence tomography assisted macular thickness profile in high myopia. Indian J Basic Appl Med Res 2015;4(2):159-166

31 Choi S, Lee S. Thickness changes in the fovea and peripapillary retinal nerve fiber layer depend on the degree of myopia. Korean J Ophthalmol. 2006; 20:215-9.

32 Marcus C. C. Lim, Sek-Tien Hoh, Paul J. Foster, Tock-Han Lim, Sek-Jin Chew, Steve K. L. Seah, Tin Aung; Use of optical coherence tomography to assess variations in macular retinal thickness in myopia. Invest. Ophthalmol. Vis. Sci. 2005;46(3):974-978

33 Lam DS, Leung KS, Mohamed S, Chan WM, Palanivelu MS, Cheung CY, et al. Regional variations in the relationship between macular thickness measurements and myopia. Invest Ophthalmol Vis Sci. 2007;48(1):376- 82. doi:10.1167/iovs.06-0426.

34 Wakitani Y, Sasoh M, Sugimoto M, et al. Macular thickness measurements in healthy subjects with different axial lengths using optical coherence tomography. Retina. 2003; 23:177-182

35 Lim MC, Hoh ST, Foster PJ, et al. Use of optical coherence tomography to assess variations in macular retinal thickness in myopia. Invest Ophthalmol Vis Sci 2005;46(3):974-978

36 Mrugacz M, Bakunowicz-Lazarczyk A, Sredzinska-Kita D. Use of optical coherence tomography in myopia. J Pediatr Ophthalmol Strabismus 2004;41(3):159-162

37 Akman A, Asena L, Güngör SG. Evaluation and comparison of the new swept source OCT-based IOL Master 700 with the IOL Master 500. Br J Ophthalmol 2016;100(9):1201-1205 


\section{Multiple Choice Questions}

1. High myopia includes:

a. Axial length more than $26 \mathrm{~mm}$.

b. Spherical equivalent more than -6 diopters (D).

c. Both a and b.

d. None of the above.

2. OCT feature with greatest correlation with myopia is:
a. Increase in perifoveal thickness.
b. Decrease in perifoveal thickness.
c. Decrease in central macular thickness.
d. Increase in parafoveal thickness.

3. Thin central macular thickness suggests:
a. Physiologic finding.
b. Due to high myopia.
c. Pathologic change.
d. Inaccurate scan.

4. Retinal nerve fiber layer thickness:

a. Temporal quadrant $>$ nasal quadrant $>$ superior quadrant $>$ inferior quadrant.

b. Inferior quadrant $>$ superior quadrant $>$ nasal quadrant $>$ temporal quadrant.

c. Superior quadrant $>$ inferior quadrant $>$ nasal quadrant $>$ temporal quadrant.

d. All are equal.

\section{Correct Answers}

1. The correct answer is option c. High myopia is defined as a refractive error of $-6.00 \mathrm{D}$ or greater and an axial length exceeding $26 \mathrm{~mm}^{4}$
2. The correct answer is option b. Whereas foveal thickness is not correlated with AL or SE, the para- and perifoveal thicknesses are negatively correlated with $\mathrm{AL}$ and, positively correlated with SE.

3. The correct answer is option c. If the macular thickness in the foveola is abnormally thin, pathologic disease should be considered. However, if changes occurred in the parafovea, other factors can first be eliminated, such as refractive factors, which could influence the measurements of macular thickness by the OCT, especially among patients with high myopia.

4. The correct answer is option b. In the previously described literature by Lee et al, Leung et al, Parvaresh et al, ad Malakar et al, ${ }^{9}$ RNFL thickness demonstrated characteristic "double hump pattern" and followed the "ISNT rule" that states that the inferior neuroretinal rim is the broadest followed by the superior, nasal, and temporal neuroretinal rim as given by Jonas et al. ${ }^{9}$ These observations are supported by histologic data as well. 